\title{
A theory for formation of a low pressure, current-free double layer
}

\author{
M A Lieberman ${ }^{1}$, C Charles and R W Boswell \\ Space Plasma, Power and Propulsion, Research School of Physical Sciences and \\ Engineering, Australian National University, ACT 0200, Australia
}

Received 29 March 2006, in final form 7 June 2006

Published 21 July 2006

Online at stacks.iop.org/JPhysD/39/3294

\begin{abstract}
A theory is developed for the formation of a low pressure, current-free double layer just inside an upstream dielectric source chamber connected to a larger diameter, downstream metallic expansion chamber. The double layer is described using four groups of charged particle: thermal ions, mono-energetic accelerated ions flowing downstream, accelerated electrons flowing upstream and thermal electrons. The accelerated electrons are formed downstream from an initially nearly half-Maxwellian electron distribution. A fifth group of counter-streaming electrons formed by the reflection of the accelerated electrons from the sheath at the insulated end wall of the source chamber is used to enforce the condition that the double layer be current-free. The condition of particle balance upstream is found to determine the double layer potential. The double layer is found to disappear at very low pressures due to loss of ionization balance upstream and due to energy relaxation processes for ionizing electrons at higher pressures. The theory is found to be in good agreement with the experiments.
\end{abstract}

(Some figures in this article are in colour only in the electronic version)

\section{Introduction}

Although double layers have been studied for decades, most theoretical papers have dealt with current-driven double layers [1]. There has been considerable interest in the formation of current-free double layers in low pressure rf-driven plasmas [2-4] and in their application to such diverse fields as plasma thrusters for space propulsion [2,5] and the physics of the solar corona [5,6]. In one recent experiment [2, 7], shown schematically in figure 1 , a plasma created by the $13.56 \mathrm{MHz}$ excitation of a helicon-type antenna wrapped around a cylindrical insulating source chamber is connected to a larger diameter metallic expansion chamber. The parameters are source length and radius $h=31 \mathrm{~cm}$ and $R_{2}=6.85 \mathrm{~cm}$ and expansion chamber length and radius $w=29.4 \mathrm{~cm}$ and $R_{1}=15.9 \mathrm{~cm}$. At argon pressures in the range $0.2-2$ mTorr, ion energy analyzer measurements show that a thin double layer is formed in the source chamber a short distance from its junction with the expansion chamber. The voltage across the double layer is measured to be as high as $\sim 25 \mathrm{~V}$ at the lowest

\footnotetext{
1 Permanent address: Department of Electrical Engineering and Computer
} Science - 1770, University of California, Berkeley, CA 94720, USA.

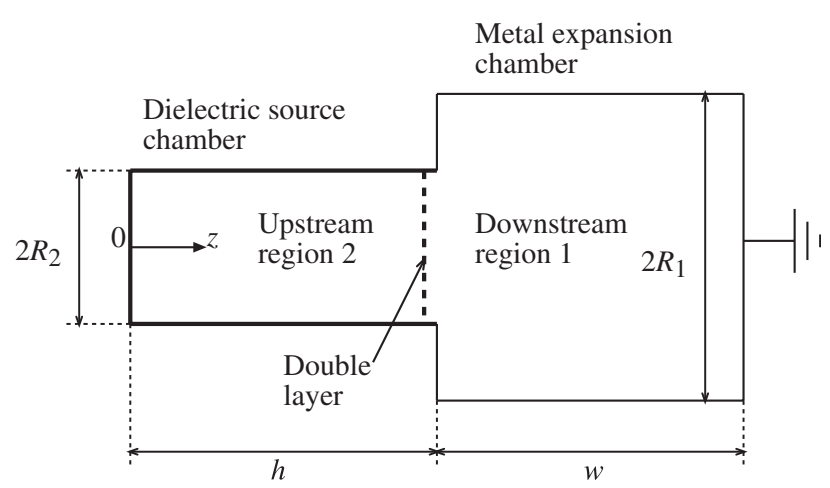

Figure 1. Schematic diagram of double layer formation at the junction between a small diameter upstream source plasma and a larger diameter downstream expansion plasma.

pressure (0.2 mTorr). For pressures below $0.2 \mathrm{mTorr}$ or above 2 mTorr, the double layer disappears.

In this work, we develop a theory that couples the dynamics of the particles in the non-neutral double layer to the diffusive flows of the quasi-neutral plasmas in the source 
and expansion chambers. The theory is found to be in good agreement with the experiments.

Langmuir [8] first described a double layer formed between an electron-emitting cathode and and ion-emitting anode, with an applied dc voltage across the gap. The space charge density is negative near the cathode and positive near the anode. Langmuir showed that the sum of positive and negative charge within the gap vanishes when

$$
I_{e}=(M / m)^{1 / 2} I_{i}
$$

where $I_{e}$ and $I_{i}$ are the electron and ion currents flowing across the gap and $m$ and $M$ are the electron and ion masses.

Andrews and Allen [9] obtained conditions to embed a double layer in a quasi-neutral plasma using four groups of charged particles inside the double layer: (a) thermal ions, (b) accelerated ions flowing downstream, (c) accelerated electrons flowing upstream and (d) thermal electrons. For assumed mono-energetic accelerated species and Maxwellian thermal species, Andrews and Allen determined the density ratios of the species at each edge of the double layer, as well as the entering velocities required for the accelerated species, at a given double layer potential drop. In section 2, we introduce an initially near-half-Maxwellian, rather than monoenergetic, accelerated electron group, to more realistically describe the experiments. To treat the formation of a currentfree double layer, as seen in the experiments, we assume that the accelerated electrons are (almost entirely) reflected from the end wall of the source region, forming a counter-streaming population.

In section 3, the densities and particle fluxes at each edge of the double layer are coupled to the particle balance relations upstream and downstream, using low pressure diffusion theory. At low pressures, the upstream and downstream thermal ionization rates are equal, but the particle loss rate is greater upstream than downstream, due to the smaller upstream radius. The accelerated electron group supplies the additional ionization rate required upstream. The ionization rate for this group depends on, and thus determines, the double layer potential. A high pressure limit is determined for the double layer formation, when the energy relaxation length for ionizing electrons $(\sim 20 \mathrm{~V})$ becomes important compared with the system length. Comparisons of the theory with the experiments are given in section 4 . We also give a discussion of some additional theoretical and experimental issues, in this section.

\section{Double layer formation}

We follow the procedure and notation of [9], with subscripts $a, b, c$ and $d$ referring to thermal ions, accelerated ions, accelerated electrons and thermal electrons, respectively, and subscripts 1 and 2 referring to the downstream and upstream double layer edges, respectively. Choosing the zero of potential at the downstream edge, with a potential $V_{s}$ at the upstream edge, the charge density within the double layer is determined by solving Poisson's equation for the potential $V$. For the thermal ions and electrons, the charge densities are given by the corresponding Boltzmann relations

$$
\rho_{a}=\rho_{a 1} \mathrm{e}^{-V / T_{i}}
$$

$$
\rho_{d}=\rho_{d 2} \mathrm{e}^{-\left(V_{s}-V\right) / T_{e}},
$$

where $\rho_{a 1}$ and $\rho_{d 2}$ are the thermal ion and electron densities at the downstream and upstream edge, respectively. In (2) and (3), and throughout this work, the symbols $T_{i}=k T_{i k} / e$ and $T_{e}=k T_{e k} / e$ are the ion and electron temperatures in equivalent-voltage units (volts), where $k=1.38 \times 10^{-23} \mathrm{~J} \mathrm{~K}^{-1}$ is Boltzmann's constant, $e=1.6 \times 10^{-19} \mathrm{C}$ is the unit of charge and $T_{i k}$ and $T_{e k}$ are the ion and electron temperatures in kelvins. For mono-energetic ions entering the double layer upstream with velocity $v_{i}$ and charge density $\rho_{b 2}$, the charge density is determined by particle flux and energy conservation within the double layer

$$
\rho_{b} v_{z}=\rho_{b 2} v_{i} ; \quad v_{z}^{2}=v_{i}^{2}+2 e\left(V_{s}-V\right) / M,
$$

which yields

$$
\rho_{b}=\rho_{b 2}\left[1+2 e\left(V_{s}-V\right) / M v_{i}^{2}\right]^{-1 / 2} .
$$

In appendix A, we treat mono-energetic accelerating electrons flowing upstream, first described in [9]. Here, we introduce a nearly-half-Maxwellian energy distribution, which more realistically describes the energy distribution seen in the experiments. At the low pressures of the experiments, the energy relaxation length for electrons with energies below the first excitation potential of argon $(\sim 11.6 \mathrm{~V})$ is much larger than the system length. Therefore, we choose the same characteristic $T_{e}$ for the accelerated group (c) as for the trapped electron group (d). As discussed in [9], the distribution function must be zero at $v_{z}=0$. We therefore introduce a near-half-Maxwellian electron distribution

$$
\begin{aligned}
& f_{c 1}=\frac{2 n_{c 1}}{\operatorname{erfc}\left(\zeta_{1}\right)}\left(\frac{m}{2 \pi e T_{e}}\right)^{1 / 2} \mathrm{e}^{-m v_{z}^{2} / 2 e T_{e}} \quad v_{z}>v_{e} \\
& =0 \quad v_{z}<v_{e}
\end{aligned}
$$

where $\zeta_{1}=\left(m v_{e}^{2} / 2 e T_{e}\right)^{1 / 2}, n_{c 1}=-\rho_{c 1} / e$, erfc is the complementary error function and the normalization is $\int f_{c 1} \mathrm{~d} v_{z}=n_{c 1}$. The velocity $v_{e}$ will be shown to be of order $\left(e T_{i} / m\right)^{1 / 2}$, which is much smaller than the electron thermal velocity for $T_{i} \ll T_{e}$. The corresponding charge density is

$$
\rho_{c}=-e \int_{v_{e}}^{\infty} v_{z}\left(v_{z}^{2}+2 e V / m\right)^{-1 / 2} f_{c 1} \mathrm{~d} v_{z} .
$$

Evaluating the integral yields

$$
\rho_{c}=\rho_{c 1} \frac{\operatorname{erfc}(\zeta)}{\operatorname{erfc}\left(\zeta_{1}\right)} \mathrm{e}^{-V / T_{e}}
$$

with $\zeta=\left[\left(m v_{e}^{2}+2 e V\right) / 2 e T_{e}\right]^{1 / 2}$.

We introduce [9] the normalized quantities $\tau_{e}=T_{e} / V_{s}$, $\tau_{i}=T_{i} / V_{s}, \eta=V / V_{s}, \Phi_{e}=m v_{e}^{2} / 2 e V_{s}$ and $\Phi_{i}=M v_{i}^{2} / 2 e V_{s}$ such that $\zeta_{1}=\left(\Phi_{e} / \tau_{e}\right)^{1 / 2}$ and $\zeta=\left[\left(\Phi_{e}+\eta\right) / \tau_{e}\right]^{1 / 2}$. The total charge density is $\rho(\eta)=\rho_{a}+\rho_{b}+\rho_{c}+\rho_{d}$.

The boundary conditions at the downstream and upstream edges are

$\rho=0 \quad$ at $\eta=0 ; \quad \rho=0 \quad$ at $\eta=1$,

$\mathrm{d} \rho / \mathrm{d} \eta=0 \quad$ at $\eta=0 ; \quad \mathrm{d} \rho / \mathrm{d} \eta=0 \quad$ at $\eta=1$.

Boundary conditions (10) are discussed in [9]; they are equivalent to the Bohm condition [10] for a collisionless 
plasma joining a collisionless sheath, such that the space derivatives of the total electron and ion densities at the sheath edge must be equal.

The four equations (9) and (10) have the form

$$
\left[\begin{array}{llll}
g_{11} & g_{12} & g_{13} & g_{14} \\
g_{21} & g_{22} & g_{23} & g_{24} \\
g_{31} & g_{32} & g_{33} & g_{34} \\
g_{41} & g_{42} & g_{43} & g_{44}
\end{array}\right]\left[\begin{array}{c}
\rho_{a 1} \\
\rho_{b 2} \\
\rho_{c 1} \\
\rho_{d 2}
\end{array}\right]=0
$$

with the coefficients

$$
\begin{aligned}
& g_{11}=1 ; \quad g_{12}=\left(1+\Phi_{i}^{-1}\right)^{-1 / 2} ; \quad g_{13}=1 ; \quad g_{14}=\mathrm{e}^{-1 / \tau_{e}} ; \\
& g_{21}=\mathrm{e}^{-1 / \tau_{i}} ; \quad g_{22}=1 ; \quad g_{23}=\mathrm{e}^{1 / \tau_{e}} \operatorname{erfc}\left(\zeta_{2}\right) / \operatorname{erfc}\left(\zeta_{1}\right) ; \\
& g_{24}=1 ; \quad g_{31}=-1 / \tau_{i} ; \quad g_{32}=\frac{1}{2} \Phi_{i}^{-1}\left(1+\Phi_{i}^{-1}\right)^{-3 / 2} ; \\
& g_{33}=\tau_{e}^{-1}\left[1-\mathrm{e}^{-\zeta_{1}^{2}} / \sqrt{\pi} \zeta_{1} \operatorname{erfc}\left(\zeta_{1}\right)\right] ; \quad g_{34}=\tau_{e}^{-1} \mathrm{e}^{-1 / \tau_{e}} ; \\
& g_{41}=-\tau_{i}^{-1} \mathrm{e}^{-1 / \tau_{i}} ; \quad g_{42}=\frac{1}{2} \Phi_{i}^{-1} ; \\
& g_{43}=\tau_{e}^{-1}\left[\mathrm{e}^{1 / \tau_{e}} \operatorname{erfc}\left(\zeta_{2}\right)-\mathrm{e}^{-\zeta_{1}^{2}} /\left(\sqrt{\pi} \zeta_{2}\right)\right] / \operatorname{erfc}\left(\zeta_{1}\right) ; \\
& g_{44}=1 / \tau_{e}
\end{aligned}
$$

with $\zeta_{2}=\left[\left(\Phi_{e}+1\right) / \tau_{e}\right]^{1 / 2}$. The determinant of the coefficient matrix must vanish for a non-zero solution of (11)

$$
\operatorname{det}[\boldsymbol{g}]=0 \text {. }
$$

The ratios of the charge densities are then given by the ratios of the cofactors of the first row of $[\mathrm{g}]$

$$
\rho_{a 1}: \rho_{b 2}: \rho_{c 1}: \rho_{d 2}:: C_{11}: C_{12}: C_{13}: C_{14}
$$

The final condition required to determine the double layer parameters is that the sum of positive and negative charge in the double layer must vanish or, equivalently, that the total force acting on the double layer must vanish:

$$
\int_{0}^{1} \rho(\eta) \mathrm{d} \eta=0 .
$$

Condition (15) ensures that a zero electric field at one edge of the double layer leads to a zero electric field at the opposite edge. Evaluating (15), we obtain

$$
E_{1} \rho_{a 1}+E_{2} \rho_{b 2}+E_{3} \rho_{c 1}+E_{4} \rho_{d 2}=0
$$

where

$$
\begin{aligned}
E_{1} & =\tau_{i}\left(1-\mathrm{e}^{-1 / \tau_{i}}\right), \quad E_{2}=2 \Phi_{i}\left[\left(1+\Phi_{i}^{-1}\right)^{1 / 2}-1\right], \\
E_{3} & =\tau_{e}\left[2\left(\zeta_{2}-\zeta_{1}\right) \mathrm{e}^{-\zeta_{1}^{2}} / \sqrt{\pi} \operatorname{erfc}\left(\zeta_{1}\right)\right. \\
& \left.+\mathrm{e}^{1 / \tau_{e}} \operatorname{erfc}\left(\zeta_{2}\right) / \operatorname{erfc}\left(\zeta_{1}\right)-1\right], \\
E_{4} & =\tau_{e}\left(1-\mathrm{e}^{-1 / \tau_{e}}\right) .
\end{aligned}
$$

Substituting (14) into (16) yields

$$
C_{11} E_{1}+C_{12} E_{2}+C_{13} E_{3}+C_{14} E_{4}=0 .
$$

For given values $\tau_{i}$ and $\tau_{e}$, the simultaneous solution of (13) and (18) determines $\Phi_{i}$ and $\Phi_{e}$. Once these are found, then
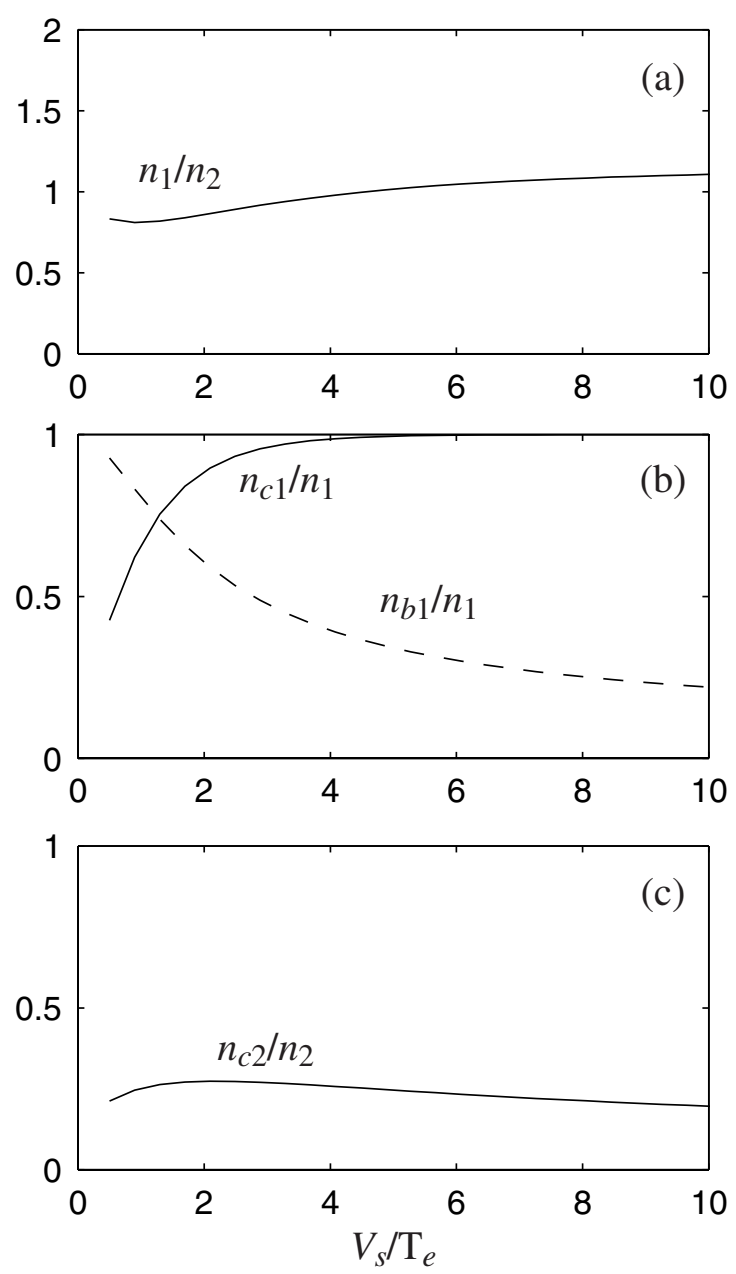

Figure 2. Density ratio versus double layer strength $V_{s} / T_{e}$; (a) $n_{1} / n_{2} ;(b) n_{c 1} / n_{1}(-)$ and $n_{b 1} / n_{1}(---)$ and (c) $n_{c 2} / n_{2}$.

the densities are obtained from (14). Except for a common constant of proportionality, these are

$n_{a 1}=C_{11} / e ; \quad n_{a 2}=g_{21} C_{11} / e ; \quad n_{b 1}=g_{12} C_{12} / e ;$

$n_{b 2}=C_{12} / e ; \quad n_{c 1}=-C_{13} / e ; \quad n_{c 2}=-g_{23} C_{13} / e ;$

$n_{d 1}=-g_{14} C_{14} / e ; \quad n_{d 2}=-C_{14} / e$.

Equations (13) and (18) are highly nonlinear and must be solved numerically. We have obtained the solutions and verified their relative accuracy to within $0.1 \%$. Figure 2(a) shows the ratio of total downstream-to-upstream density $n_{1} / n_{2}$ as a function of the double layer strength $V_{s} / T_{e}$, for an assumed ion-to-electron temperature ratio $T_{i} / T_{e}=0.05$. Here $n_{1}=n_{a 1}+n_{b 1}$ and $n_{2}=n_{a 2}+n_{b 2}$. The density ratio is nearly unity over the entire range of strengths. Figure $2(b)$ gives the densities for the accelerated electron group (solid line) and the accelerated ion group (dashed line) as fractions of the total downstream density. The electron group rises sharply to $100 \%$ of the total as $V_{s} / T_{e}$ increases, and the ion group falls from nearly $100 \%$ to $20 \%$ over the range of $V_{s} / T_{e}$ shown. The electron group is about $90 \%$ of the total downstream density and the ion group is about $55 \%$, for $V_{s} / T_{e}=2$. Figure $2(c)$ gives the density of the accelerated electron group as a fraction of the total upstream density. The 

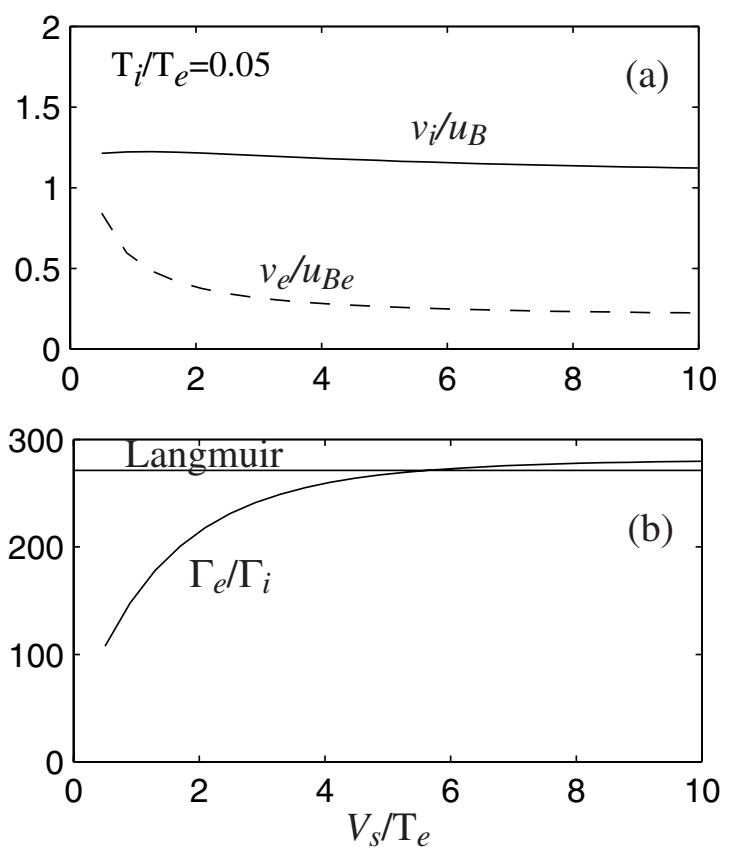

Figure 3. (a) Ion velocity $v_{i}(-)$ and electron velocity $v_{e}(----)$ entering the double layer versus $V_{s} / T_{e}$; the ion velocity is normalized to $u_{B}=\left(e T_{e} / M\right)^{1 / 2}$ and the electron velocity to $u_{B e}=\left(e T_{i} / m\right)^{1 / 2} ;(b)$ the ratio of electron-to-ion flux for the accelerated species versus $V_{s} / T_{e}$; the Langmuir condition (1) is shown as the solid horizontal line.

accelerated electrons comprise about $20 \%$ of the upstream density, relatively independent of the ratio $V_{s} / T_{e}$. There is a negligible thermal ion density $n_{a 2}$ passing from downstream to upstream. Figure $3(a)$ gives the ratios $v_{i} / u_{B}$ (solid line) and $v_{e} / u_{B e}$ (dashed line), where $u_{B}=\left(e T_{e} / M\right)^{1 / 2}$ is the usual Bohm velocity and $u_{B e}=\left(e T_{i} / m\right)^{1 / 2}$ is a similar velocity defined for electrons. The accelerating ion group is seen to enter the double layer at a velocity of $1.2-1.3 u_{B}$. The entering velocity $v_{e}$ for the lowest energy electrons in the accelerating electron distribution is seen to be in the range $0.2-1 u_{B e}$, which is small compared with $\left(e T_{e} / m\right)^{1 / 2}$. These results are found to be practically independent of $T_{i} / T_{e}$ over the range $0.01-$ 0.06 , except that $v_{e}$ decreases as $T_{i} / T_{e}$ decreases. The ratio of electron-to-ion flux for the accelerating species

$$
\Gamma_{e} / \Gamma_{i}=\frac{n_{c 1}}{n_{b 2}} \frac{u_{B}}{v_{i}} \frac{\mathrm{e}^{-\zeta_{1}^{2}}}{\operatorname{erfc}\left(\zeta_{1}\right)}\left(\frac{2 M}{\pi m}\right)^{1 / 2}
$$

is compared with the Langmuir ratio (1) in figure 3(b). At low double layer strengths, we see a significant reduction compared with the Langmuir ratio.

The entire source chamber is insulating in the experiments, such that the net (electrical) current flowing into the source must vanish. To apply the preceding results to this situation, a fifth species is introduced, which is formed by the reflection of (almost) all the accelerated electron group by the sheath at the upstream wall. It is easily seen that this reflected group contributes exactly the same charge density to the double layer and to the downstream plasma as the original group. Hence, the double layer solution is unaltered by the addition of the reflected electron group. In this sense, there is no fundamental distinction between a current-carrying and a
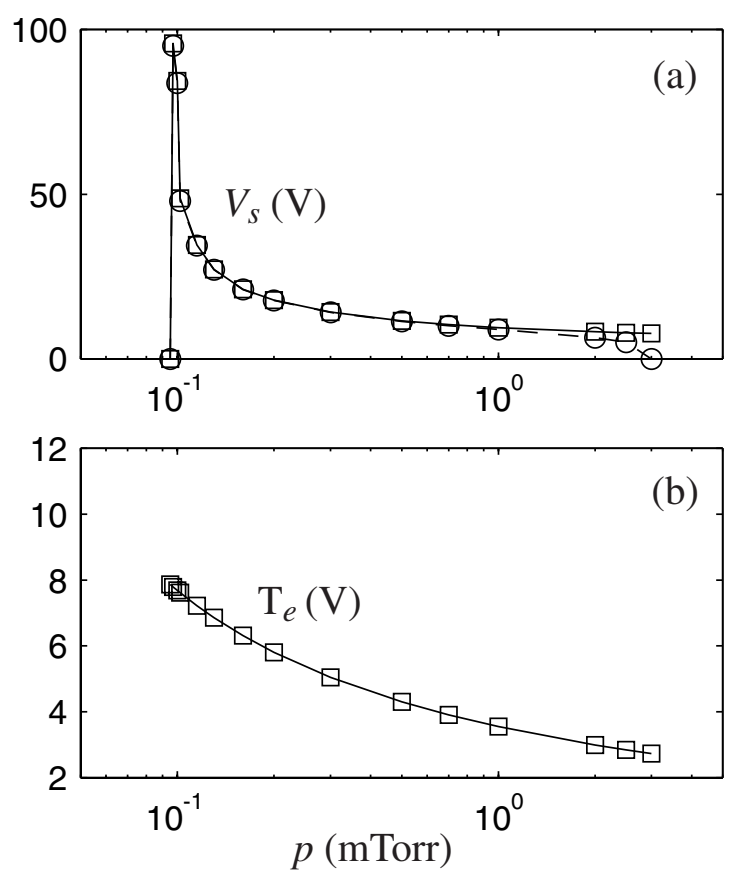

Figure 4. (a) Double layer strength $V_{s}$ versus pressure $p$ without $(\square)$ and with $(O)$ accounting for the energy relaxation length of ionizing electrons; $(b)$ electron temperature $T_{e}$ versus pressure $p$; the lines are drawn to guide the eye.

current-free double layer [11]. The slight imbalance in currents between the incident and reflected accelerated and thermal electron groups balances the flow of ions to the upstream wall. The resulting floating potential that forms at the wall will be determined in section 3 .

\section{Downstream and upstream particle balance}

The downstream particle balance is determined by low pressure diffusion theory [12, section 10.2] according to the relation

$$
K_{\mathrm{iz}} n_{g} \pi R_{1}^{2} \cdot 2 w=\left(2 \pi R_{1}^{2} h_{2 w}+2 \pi R_{1} \cdot 2 w h_{R_{1}}\right) u_{B},
$$

where $K_{\mathrm{iz}}\left(T_{e}\right)$ is the ionization rate coefficient for thermal (Maxwellian) electrons, $n_{g}$ is the neutral gas density,

$h_{2 w}=0.86\left(3+w / \lambda_{i}\right)^{-1 / 2}, \quad h_{R_{1}}=0.8\left(4+R_{1} / \lambda_{i}\right)^{-1 / 2}$

are the axial and radial edge-to-centre density ratios, given from low pressure diffusion theory [12, p 148] and $\lambda_{i} \approx$ $(330 p)^{-1}$ is the thermal ion mean free path in argon, with $\lambda_{i}$ in $\mathrm{cm}$ and $p$ in Torr. Solving (21) determines $T_{e}$ in the entire system at any given pressure. Guided by the experiments and the results from the double layer theory, we have assumed in (21) and (22) that the thermal ion diffusion flux flowing upstream into the source chamber is negligible.

Because the upstream radius is smaller than the downstream radius, ionization by thermal electrons upstream does not suffice to balance the larger particle losses upstream at low pressures. An additional source of upstream ionization is required, which is supplied by the accelerated group of electrons. We use a simplified one-dimensional diffusion model for the particle balance upstream. Letting $n_{i}$, 
$n_{d}=n_{i}-n_{c 2}$ and $n_{c 2}$ be the densities of ions, thermal electrons and accelerated electrons, with the latter species assumed to be uniform in $z$, then the diffusion equation is

$$
-D_{A 2} \frac{\mathrm{d}^{2} n_{i}}{\mathrm{~d} z^{2}}+v_{R} n_{i}=n_{g}\left(K_{\mathrm{iz}} n_{d}+K_{\mathrm{izc}} n_{c 2}\right),
$$

where

$$
D_{A 2}=h_{R_{2}} u_{B} R_{2}
$$

is the low pressure ambipolar diffusion coefficient (see appendix B),

$$
v_{R}=2 h_{R_{2}} u_{B} / R_{2}
$$

is the radial loss frequency and $K_{\mathrm{izc}}$ is the ionization rate coefficient for the accelerated electron group. $K_{\text {izc }}$ is a function of $V_{s}$ and $T_{e}$ and is calculated in appendix C. We assume a symmetric solution for the ion density about $z=h / 2$

$$
n_{i}=\beta n_{c 2}-n_{i 0} \cosh \gamma\left(z-\frac{1}{2} h\right),
$$

where

$$
\beta=\frac{n_{g}\left(K_{\mathrm{izc}}-K_{\mathrm{iz}}\right)}{\left(v_{R}-K_{\mathrm{iz}} n_{g}\right)}
$$

and

$$
\gamma^{2}=\left(v_{R}-K_{\mathrm{iz}} n_{g}\right) / D_{A 2}
$$

is the square of the axial decay constant. The ion flux is

$$
\Gamma_{i}=\gamma D_{A 2} n_{i 0} \sinh \gamma\left(z-\frac{1}{2} h\right) .
$$

Setting $\Gamma_{i}=n_{i} v_{i}$ at $z=h$ determines $n_{i 0}$. The complete solution is then

$$
n_{i}=\left[1-\frac{\cosh \gamma\left(z-\frac{1}{2} h\right)}{\left(\gamma D_{A 2} / v_{i}\right) \sinh \frac{1}{2} \gamma h+\cosh \frac{1}{2} \gamma h}\right] \beta n_{c 2} .
$$

Evaluating (30) at the upstream edge of the double layer $z=h$ yields

$$
\beta=\left(1+\frac{v_{i}}{\gamma D_{A 2}} \operatorname{coth} \frac{1}{2} \gamma h\right) \frac{n_{i 2}}{n_{c 2}},
$$

where $n_{i 2}=n_{a 2}+n_{b 2}$ is the total ion density at the upstream edge. The ratio $n_{i 2} / n_{c 2}$ in (31) is given from (19). Equating (27) to (31) yields

$$
\frac{n_{g}\left(K_{\mathrm{izc}}-K_{\mathrm{iz}}\right)}{\left(v_{R}-K_{\mathrm{iz}} n_{g}\right)}=\left(1+\frac{v_{i}}{\gamma D_{A 2}} \operatorname{coth} \frac{1}{2} \gamma h\right) \frac{n_{i 2}}{n_{c 2}} .
$$

Since $K_{\mathrm{izc}}$ and $n_{i 2} / n_{c 2}$ depend on $V_{s}$, (32) determines the double layer strength $V_{s}$ needed for particle balance upstream.

Equation (32) has been solved numerically, and the results for $V_{s}$ versus pressure $p$ are given as the squares in figure 4, along with $T_{e}$ determined by solving (21). We see that $V_{s}$ rises dramatically as the pressure is decreased, with a minimum pressure of approximately 0.096 mTorr for a solution to exist. Below that pressure, the maximum ionization rate coefficient of $\sim 2.2 \times 10^{-13} \mathrm{~m}^{3} \mathrm{~s}^{-1}$ for the accelerated electrons upstream (see figure $\mathrm{C} 1$ ) is not sufficient to balance the excess upstream particle losses. The maximum double layer strength is seen to be of the order $100 \mathrm{~V}$ near the minimum pressure but is a very sensitive function of pressure for such high values of $V_{s}$. Figure 5 shows the various density ratios corresponding
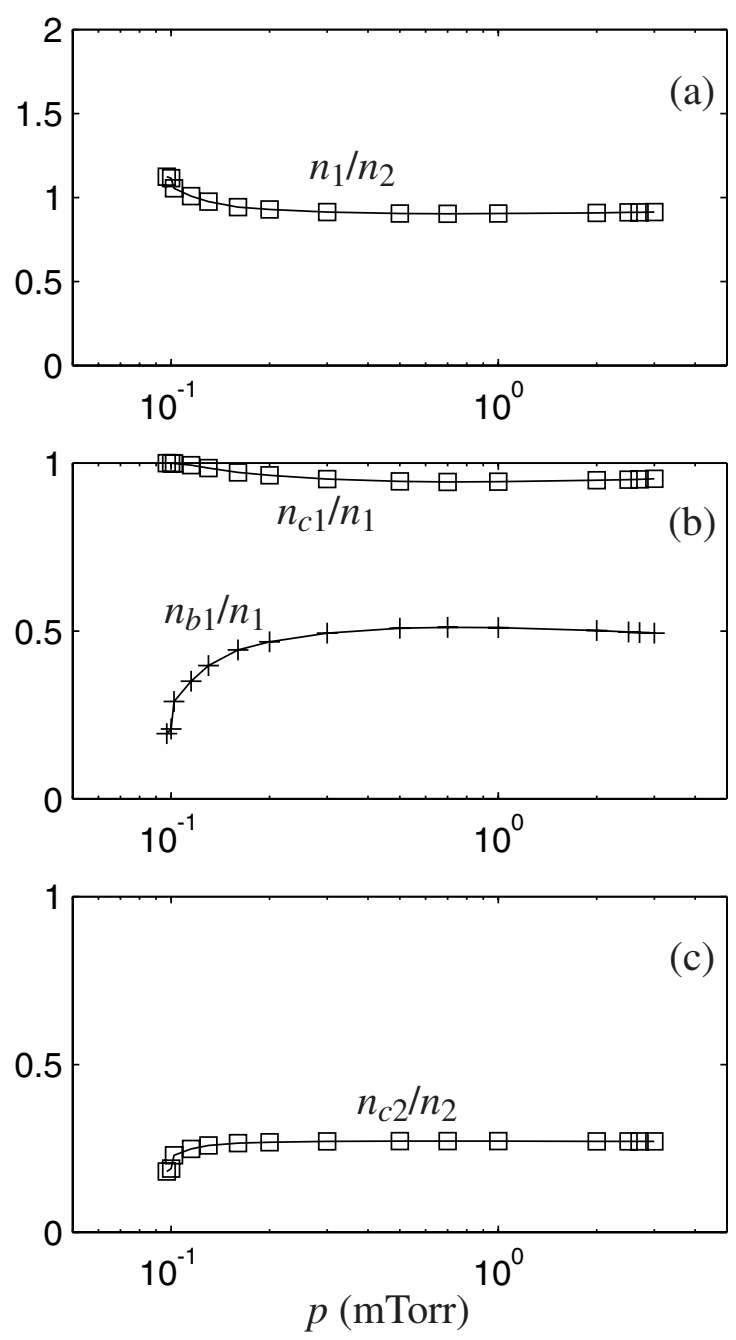

Figure 5. (a) Downstream-to-upstream density ratio $n_{1} / n_{2}$,

(b) downstream ratios $n_{c 1} / n_{1}$ and $n_{b 1} / n_{1}$ and (c) upstream ratio $n_{c 2} / n_{2}$ versus pressure $p$ for the solution shown in figure 4 ; the lines are drawn to guide the eye.

to figure 4. These have little variations with pressure except at the lowest pressures.

In the experiment, the double layer also disappears at pressures above a few milliTorr. To understand this, we examine the energy relaxation length for ionizing electrons [12, p 691],

$$
\lambda_{\mathrm{iz}}=\left(\lambda_{\mathrm{el}} \lambda_{\mathrm{inel}} / 3\right)^{1 / 2},
$$

where $\lambda_{\text {el }}$ and $\lambda_{\text {inel }}$ are the elastic and inelastic mean free paths for electrons in argon. For a $20 \mathrm{~V}$ electron, we find that $\lambda_{\mathrm{iz}} \approx 30 \mathrm{~cm}$ at $1 \mathrm{mTorr}$, which is comparable to the system length. Since electrons are heated upstream, the downstream ionizing electron density can be depleted at the higher pressures. When the ratio of downstream-to-upstream ionization rates becomes equal to the ratio of downstreamto-upstream particle loss rates, then the additional ionization provided by electrons accelerated upstream by the double layer is no longer needed. For a uniform ionizing density $n_{\mathrm{iz} 2}$ upstream, the downstream density is

$n_{\mathrm{iz} 1}=n_{\mathrm{iz} 2} \cosh \left[(h+w-z) / \lambda_{\mathrm{iz}}\right] / \cosh \left(w / \lambda_{i z}\right) ;$

$$
h<z<h+w \text {, }
$$


where we have assumed electron reflection from the downstream sheath. The average downstream density is

$$
\bar{n}_{\mathrm{iz} 1}=\frac{1}{w} \int_{h}^{h+w} \mathrm{~d} z n_{\mathrm{iz} 1}=n_{\mathrm{iz} 2} \frac{\lambda_{\mathrm{iz}}}{w} \tanh \frac{w}{\lambda_{\mathrm{iz}}} .
$$

Hence, for a given downstream ionization rate coefficient $K_{\text {iz }}$, the effective ionization rate coefficient upstream is larger and is given by

$$
K_{\mathrm{izeff}}=K_{\mathrm{iz}} \frac{w}{\lambda_{\mathrm{iz}}} \operatorname{coth} \frac{w}{\lambda_{\mathrm{iz}}} .
$$

Using (36) in place of $K_{\mathrm{iz}}$ in (32), we can solve to determine $V_{s}$, as previously. The results are shown in figure 4 as circles. We find that the double layer disappears at pressures above about $2.5 \mathrm{mTorr}$, as is seen experimentally.

Downstream, the accelerated ions enter the expansion chamber, where they undergo charge transfer collisions with the neutral gas, leading to the production of thermal ions. The experiments show that the characteristic radius of the beam ions inside the expansion chamber is that of the source chamber. The neutralizing electrons associated with the downstream beam ions also lead to additional ionization. A simplified diffusion model is used to describe these processes, in which the ionization and the volume rate of production of thermal ions due to the presence of the beam ions is assumed to be uniform within the expansion chamber. The downstream beam ion density is

$n_{b}=n_{b 1} \mathrm{e}^{-(z-h) / \lambda_{b}} ; \quad h<z<h+w ; \quad r<R_{2}$,

where $\lambda_{b} \approx 0.7 \lambda_{i}$ is the beam ion-neutral charge transfer mean free path. The net number of beam ions per second converted to thermal ions in the expansion chamber is thus

$$
n_{b 1} v_{b}\left(1-\mathrm{e}^{-w / \lambda_{b}}\right) \pi R_{2}^{2}
$$

where $v_{b}=\left(v_{i}^{2}+2 e V_{s} / M\right)^{1 / 2}$ is the beam ion velocity in the expansion chamber. Dividing by the volume of the expansion chamber, the volume rate of production of thermal ions is then $v_{b} n_{b \text { eff }}$, where $v_{b}=v_{b} / \lambda_{b}$ is the charge transfer frequency and

$$
n_{b \text { eff }}=n_{b 1} \frac{\lambda_{b}}{w} \frac{R_{2}^{2}}{R_{1}^{2}}\left(1-\mathrm{e}^{-w / \lambda_{b}}\right) .
$$

The diffusion equation for thermal ions downstream is

$$
-D_{A 1} \frac{\mathrm{d}^{2} n_{i}}{\mathrm{~d} z^{2}}+v_{R_{1}} n_{i}=n_{g} K_{\mathrm{iz}}\left(n_{i}+n_{b \text { eff }}\right)+v_{b} n_{b \text { eff }},
$$

where $D_{A 1}=h_{R_{1}} u_{B} R_{1}$ (see appendix B) and $v_{R_{1}}=$ $2 h_{R_{1}} u_{B} / R_{1}$ is the downstream radial loss frequency. The solution is

$$
n_{i}=n_{a 1} \cos k_{1}(z-h)+\alpha n_{b \mathrm{eff}}\left[\cos k_{1}(z-h)-1\right],
$$

where

$$
k_{1}^{2}=\left(n_{g} K_{\mathrm{iz}}-v_{R_{1}}\right) / D_{A 1}
$$

is the square of the downstream axial wavenumber and

$$
\alpha=\frac{k_{1}^{2} D_{A 1}+v_{R_{1}}+v_{b}}{k_{1}^{2} D_{A 1}} .
$$

The thermal ion flux is

$$
\Gamma_{i}=k_{1} D_{A 1}\left(n_{a 1}+\alpha n_{b \mathrm{eff}}\right) \sin k_{1}(z-h) .
$$

Setting $\Gamma_{i}=n_{i} u_{B}$ at $z=h+w$ yields

$k_{1} D_{A 1}\left(n_{a 1}+\alpha n_{b e f f}\right) \sin k_{1} w$

$$
=u_{B}\left(n_{a 1}+\alpha n_{b \text { eff }}\right) \cos k_{1} w-u_{B} \alpha n_{b \text { eff }} \text {. }
$$

Solving (45) for $k_{1}$, we obtain the density profile from (40); $k_{1}$ is found to be real at low pressures and imaginary at higher pressures.

The potential distribution in the discharge is determined from the density distribution. With the zero of potential at the downstream wall and a floating potential of $V_{f 1}=$ $\frac{1}{2} T_{e} \ln (M / 2 \pi m)=4.8 T_{e}$ across the sheath there, the downstream potential is

$$
V_{1}(z)=4.8 T_{e}+T_{e} \ln \left(n_{e}(z) / n_{e}(h+w)\right) .
$$

The upstream potential is

$$
V_{2}(z)=V_{1}(h)+V_{s}+T_{e} \ln \left(n_{d 2}(z) / n_{d 2}(h)\right) .
$$

The potential drop $V_{f 2}$ across the sheath at the upstream floating wall is obtained by equating the ion and electron fluxes there

$$
n_{i 2} v_{i}=\frac{1}{4}\left(\frac{8 e T_{e}}{\pi m}\right)^{1 / 2}\left(n_{d 2}+n_{c 1} \mathrm{e}^{V_{s} / T_{e}}\right) \mathrm{e}^{-V_{f 2} / T_{e}} .
$$

Figure 6 shows the density and potential profiles at low ( 0.1 mTorr), medium ( 0.3 mTorr) and high ( 1 mTorr) pressures. The potentials (with respect to earth) at the upstream and downstream walls are indicated by crosses. At low pressures, a large double layer potential drop $V_{s}$ is seen, and the charge transfer of the ion beam with the neutrals is small. The medium and high pressure cases are similar, with a lower double layer potential and almost complete charge transfer of the ion beam.

\section{Comparison of theory with measurements}

In a relatively complete experiment in argon gas with source length and radius $h=31 \mathrm{~cm}$ and $R_{2}=6.85 \mathrm{~cm}$ and expansion chamber length and radius $w=29.4 \mathrm{~cm}$ and $R_{1}=15.9 \mathrm{~cm}$, the double layer potential $V_{s}$ was measured as a function of the pressure $p$. The measurements are shown in figure 7 as the solid squares, with the theoretical curve shown as a solid line. The general shape and height of the theoretical curve match the experimental results quite well. We see experimentally the low and high pressure cutoffs for double layer formation, as also seen in the theory. At low pressures, the experimental pressure measurement is estimated to have an accuracy of about 0.1 mTorr. Figure 8 shows the theory results for the potential and the thermal ion density at $0.2 \mathrm{mTorr}$ (left side), for comparison with the experimental result published in [2] (right side). The thermal ion density was measured using a retarding potential energy analyzer whose orifice was oriented at right angles to the exiting ion beam. The general shapes of the potential and the thermal ion density are quite similar to that seen experimentally. The theoretical densities agree quite well with the measurements on both sides of the double layer. A somewhat smaller double layer potential $V_{s}$ 

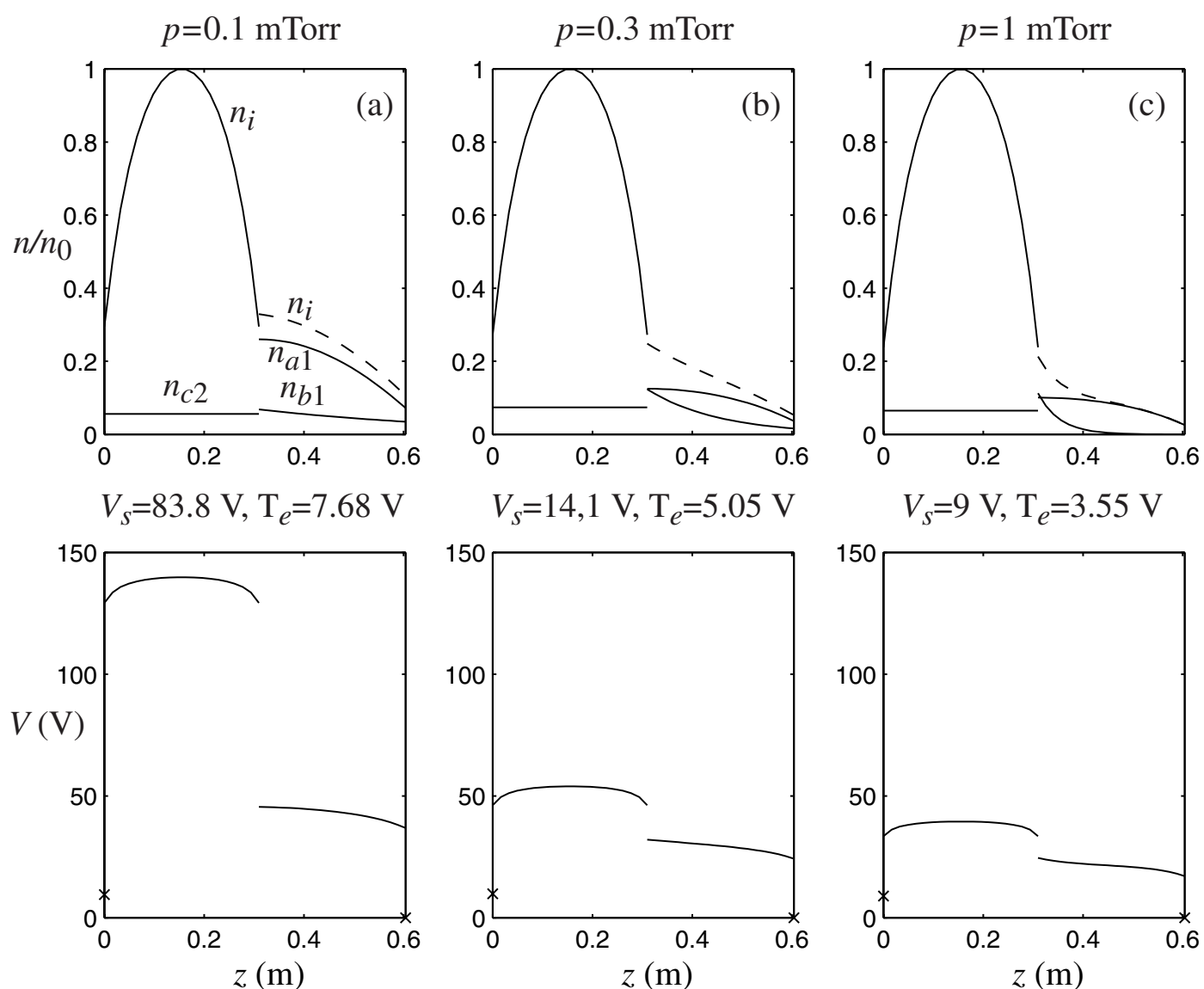

Figure 6. Potential $V$ and total ion density $n_{i}$ versus position $z$ at $(a) 0.1 \mathrm{mTorr},(b) 0.3 \mathrm{mTorr}$ and $(c) 1 \mathrm{mTorr}$; the downstream total density $(---)$ is decomposed into the accelerated ion beam density (decaying exponential, $\longrightarrow$ ) and the thermal ion density $(-$ ); the wall potentials are given by the crosses $(\times)$.

is seen in the theory than in the experiment. However, $V_{s}$ is a quite sensitive function of pressure in this range, and a small decrease in the theoretical pressure will give a better match to the experimental result for $V_{s}$. An unexplained feature of the experimental results, not seen in the theory, is the large potential rise upstream of the double layer, which may be an artefact of the experimental measurement for $z$ less than $0.2 \mathrm{~m}$, where a non-negligible earthed area (probe shaft) is introduced into the insulated region 2 [7]. Figure 9 shows the theoretical results for the decay of the relative ion beam density versus position at 0.27 mTorr argon. The measured beam decay (solid squares) is somewhat faster than the theoretical decay (solid line) given by (37), but the results are quite sensitive to the uncertainty in the pressure measurement and to the value $\lambda_{b} \approx 0.7 \lambda_{i}$ used in the theory.

In another experiment in a larger device, the double layer potential was measured over a somewhat limited range of pressures in argon [13]. The experimental parameters were source length and radius $h=57.8 \mathrm{~cm}$ and $R_{2}=10 \mathrm{~cm}$ and expansion chamber length and radius $w=200 \mathrm{~cm}$ and $R_{1}=$ $50 \mathrm{~cm}$. The experimental results are shown as solid squares in figure 10, with the theoretical curve shown as the solid line. There is a reasonable agreement between the measurements and the theory, although the full range of pressures was not explored in the measurements.

In this work we have developed a theory for the formation of a low pressure current-free double layer in

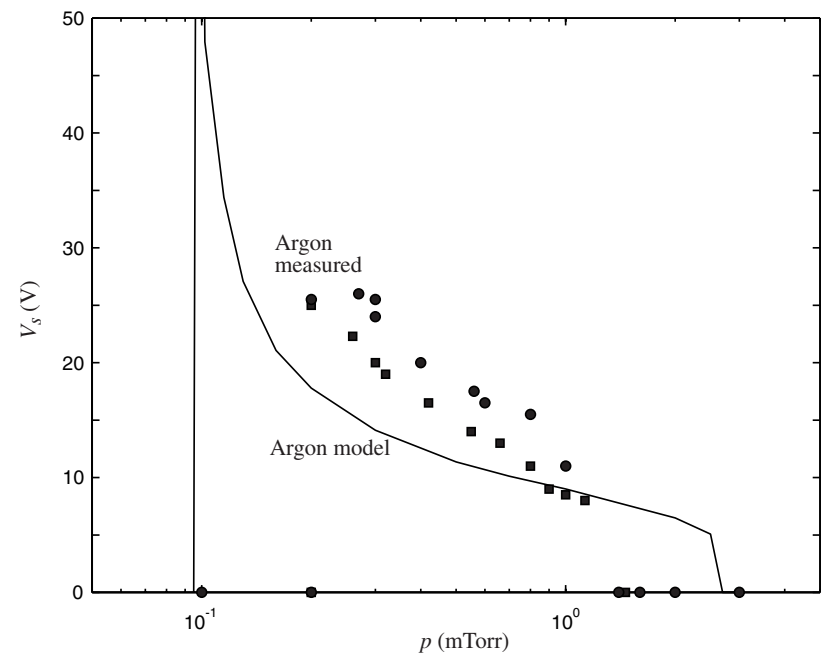

Figure 7. Double layer potential $V_{s}$ versus pressure $p$; measurements from 1999 in argon (ם) [15] and from 2006 (•) using two different energy analysers; theory for argon (-

a plasma expanding from a small diameter source to a larger diameter chamber. The diffusive flows of the quasineutral plasmas in the source and expansion chamber were coupled to the dynamics of the particles in the non-neutral double layer. To preserve the current-free nature of the 
Theory for formation of a low pressure, current-free double layer
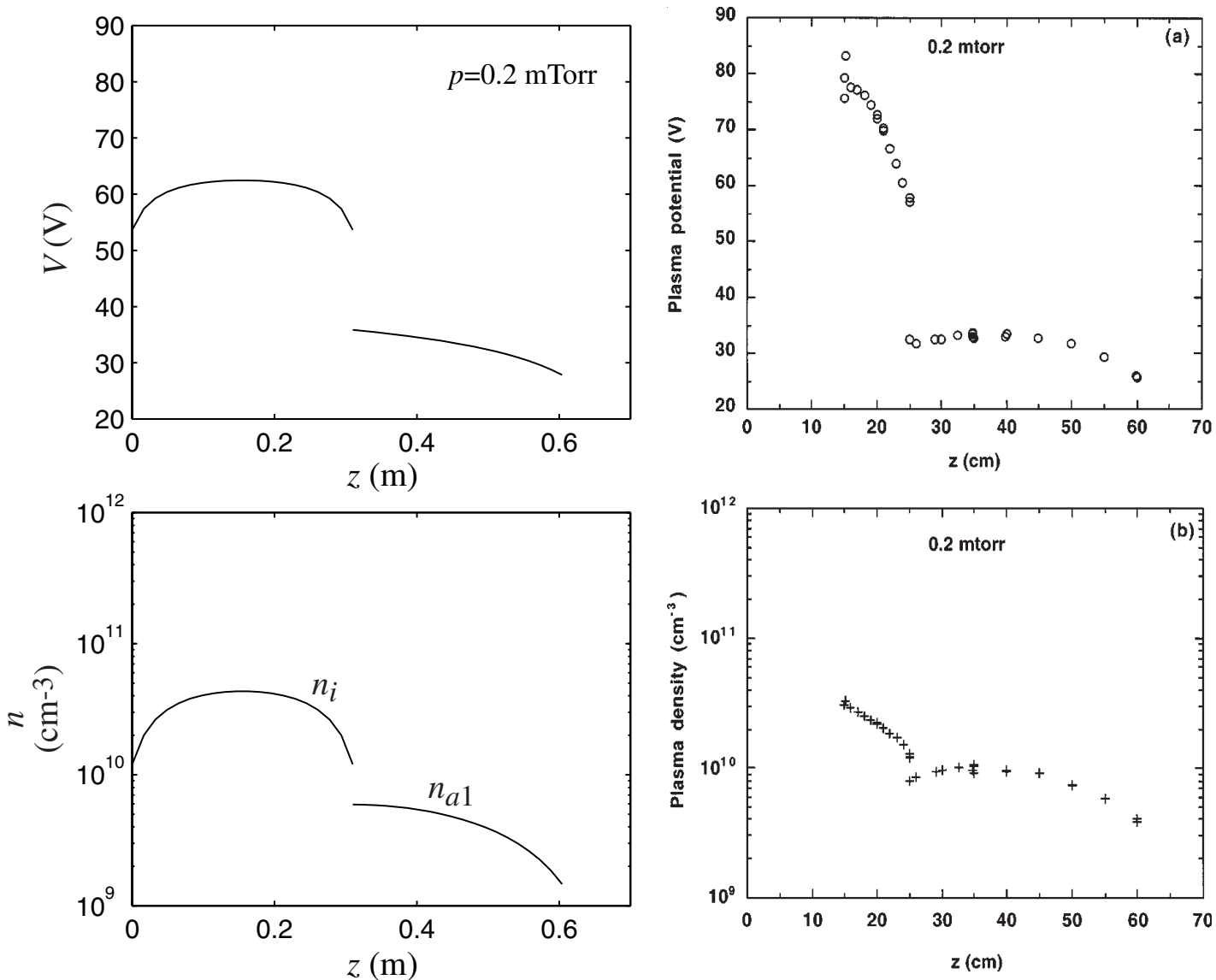

Figure 8. Potential $V$ (top panes) and thermal ion density $n_{a}$ (bottom panes) versus position $z$ at 0.2 mTorr; the left-hand panes give the model results and right-hand panes give the experimental results in [7].

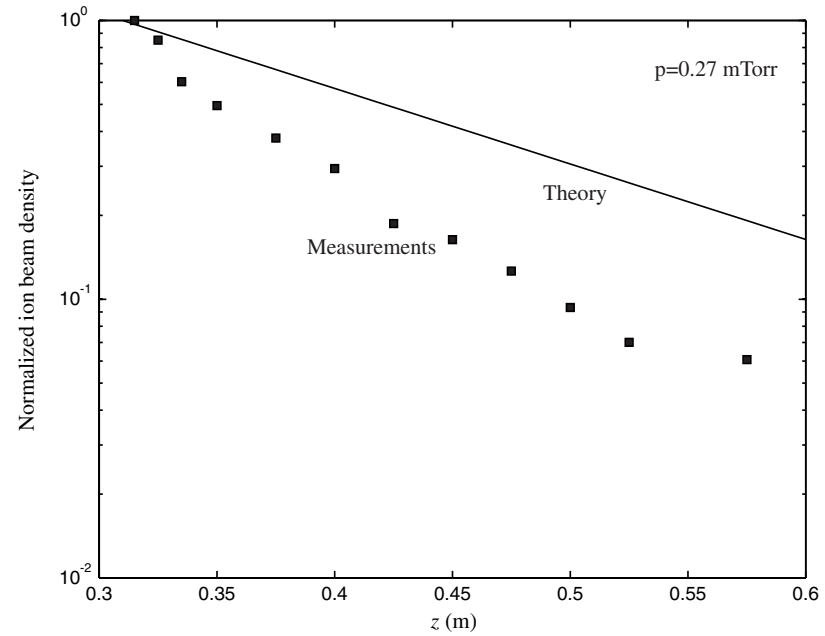

Figure 9. Relative ion beam density versus position $z$; measurements in argon ( $\square)$; theory for $\operatorname{argon}(-)$.

experimental double layer, a fifth species consisting of a counter-streaming population of electrons formed by the reflection of the accelerated electrons from the end wall of the source region has been introduced. The results of the theory are in reasonable agreement with experimental results obtained on two different-sized devices exhibiting double layer formation.

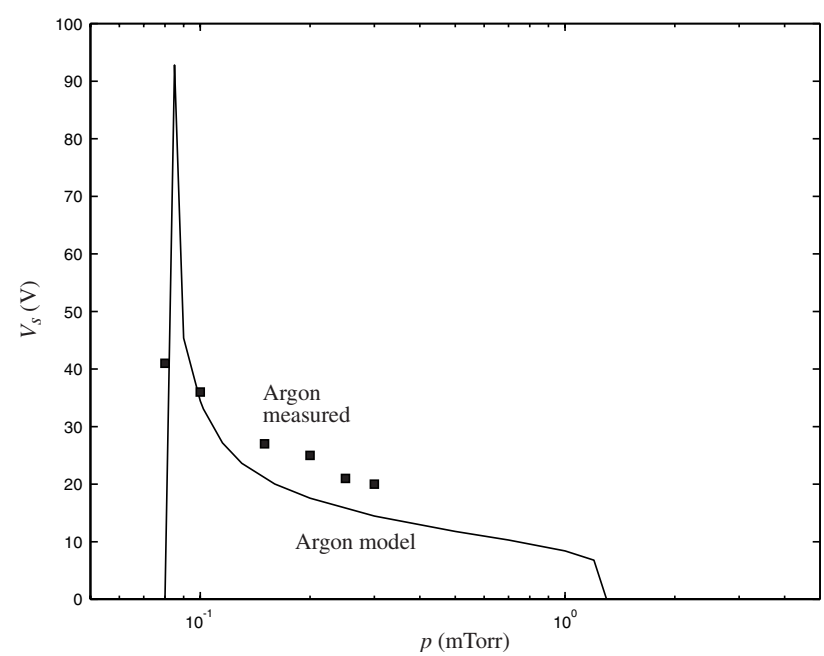

Figure 10. Double layer potential $V_{s}$ versus pressure $p$ in a larger device; measurements in argon ( $\boldsymbol{\square})$ from [13]; theory for argon $(-)$.

\section{Acknowledgments}

MAL acknowledges the support provided by the Lam Research Corporation, the State of California MICRO Program, National Science Foundation Grant ECS-0139956, a UC Discovery Grant from the Industry-University Cooperative Research 
Program (IUCRP) and the SP3 group at ANU. We thank A Viquerat for his assistance with initial modelling studies. MAL thanks A J Lichtenberg for enlightening discussions on double layer physics.

\section{Appendix A. Monoenergetic accelerated electrons}

Double layer formation with mono-energetic accelerated electrons was examined in [9]. The modified terms in (12) and (17) are

$g_{23}=\left(1+\Phi_{e}^{-1}\right)^{-1 / 2} ; \quad g_{33}=-\left(2 \Phi_{e}\right)^{-1} ;$

$g_{43}=-\left(2 \Phi_{e}\right)^{-1}\left(1+\Phi_{e}^{-1}\right)^{-3 / 2}$

and

$$
E_{3}=2 \Phi_{e}\left[\left(1+\Phi_{e}^{-1}\right)^{1 / 2}-1\right]
$$

In [9] additional approximations were made that the thermal ion density upstream and the thermal electron density downstream were negligible

$$
g_{14}=g_{21}=g_{34}=g_{41}=0 ; \quad E_{1}=\tau_{i} ; \quad E_{4}=\tau_{e} .
$$

Using these assumptions, (13) and (18) were solved, yielding the solutions plotted in [9]. (There are typographical sign errors in [9] for $g_{33}, g_{41}$ and $g_{43}$, which we have corrected here.) An interesting feature is that, due to approximation (A3), solutions do not exist for certain ranges of $\tau_{e}$ and $\tau_{i}$; for example, solutions do not exist for $\tau_{e} \gtrsim 0.4$ at $\tau_{i} \gtrsim 0.04$ (see figure 2 of [9]). For small double layer strengths, with $V_{s} \lesssim$ few $T_{e}$, the thermal electron density downstream is significant. Solving (13) and (18) when the approximation (A3) is not made yields the results for $\Phi_{i}$ and $\Phi_{e}$ shown in figure A1.

\section{Appendix B. Low pressure diffusion coefficient}

For $R_{2} \ll h$ in the pressure regime $T_{i} / T_{e} \ll \lambda_{i} / R_{2} \ll 1$, an estimate for the ambipolar diffusion coefficient $D_{A 2}$ is

$$
D_{A 2}=\mu_{i} T_{e}
$$

where

$$
\mu_{i}=\left(2 e \lambda_{i} / \pi M E\right)^{1 / 2}
$$

is the low pressure mobility [12, p 144]. The radial ambipolar electric field is the strongest component and is estimated as

$$
E=-T_{e}\left(\frac{1}{n} \frac{\mathrm{d} n}{\mathrm{~d} r}\right) \approx \frac{T_{e}}{R_{2}} .
$$

Substituting (B2) and (B3) into (B1) yields

$$
D_{A 2} \approx 0.8\left(\lambda_{i} / R_{2}\right)^{1 / 2} u_{B} R_{2} .
$$

An extension of (B4) to the collisionless (Langmuir) pressure regime $\lambda_{i} \gg R_{2}$ is obtained using the edge-to-centre density ratio $h_{R_{2}}$, through the substitution in (B4) that

$$
0.8\left(\lambda_{i} / R_{2}\right)^{1 / 2} \longrightarrow h_{R_{2}}
$$

with

$$
h_{R_{2}}=0.8 /\left(4+R_{2} / \lambda_{i}\right)^{1 / 2}
$$
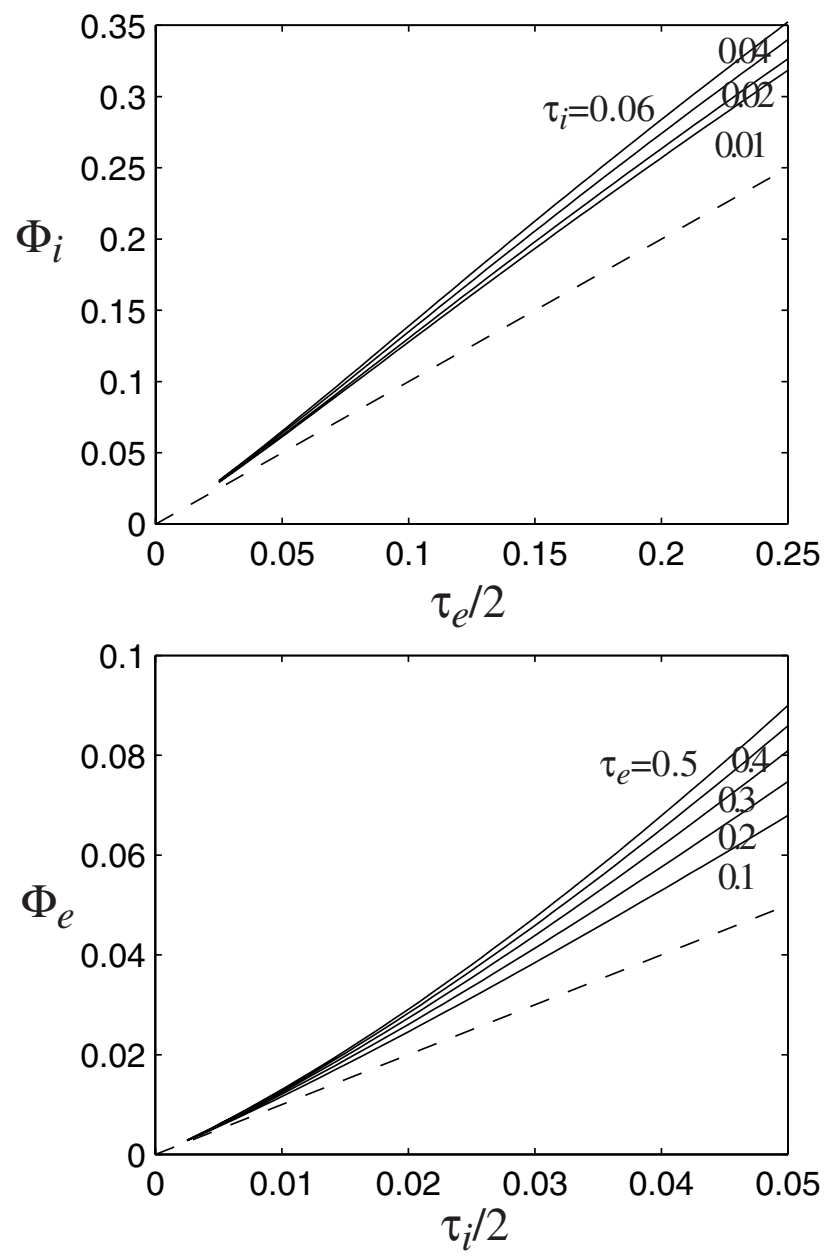

Figure A1. Double layer formation for mono-energetic accelerated electrons and ions; the approximation of negligible thermal ion density upstream and thermal electron density downstream is not made; (a) $\Phi_{i}$ versus $\tau_{e} / 2$ for various values of $\tau_{i} ;(b) \Phi_{e}$ versus $\tau_{i} / 2$ for various values of $\tau_{e}$.

as in (21). The final result is

$$
D_{A 2} \approx h_{R_{2}} u_{B} R_{2} \text {. }
$$

\section{Appendix C. Ionization rate coefficient for accelerated electrons}

We calculate the rate coefficient $K_{\text {izc }}$ for accelerated electrons upstream, which depends on both $T_{e}$ and $V_{s}$. The accelerated electron flux is conserved across the double layer

$$
v_{z 2} d v_{z 2} f_{c 2}=v_{z 1} d v_{z 1} f_{c 1}
$$

with

$$
v_{z 2}^{2}=v_{z 1}^{2}+2 e V_{s} / m
$$

Since $v_{z 2} d v_{z 2}=v_{z 1} d v_{z 1}$, the distribution function is conserved across the double layer. Hence, $f_{c 2}\left(v_{z 2}\right)=$ $f_{c 1}\left(v_{z 2}\left(v_{z 1}\right)\right)$. Using $f_{c 1}$ from (6), along with its counterstreaming counterpart, and ignoring the small 'hole' in the 
Theory for formation of a low pressure, current-free double layer

distribution for $\left|v_{z 1}\right|<v_{e}$, we obtain

$f_{c 2}=n_{c 1}\left(\frac{m}{2 \pi e T_{e}}\right)^{3 / 2} \mathrm{e}^{V_{s} / T_{e}} \mathrm{e}^{-m v_{2}^{2} / 2 e T_{e}} ; \quad\left|v_{z 2}\right|>v_{s}$ $=0 \quad$ otherwise

with $v_{s}=\left(2 e V_{s} / m\right)^{1 / 2}$ and with $v_{2}^{2}=v_{x 2}^{2}+v_{y 2}^{2}+v_{z 2}^{2}$. The upstream density is

$$
n_{c 2}=\int f_{c 2} \mathrm{~d}^{3} v_{2}=n_{c 1} \mathrm{e}^{V_{s} / T_{e}} \operatorname{erfc}\left(\zeta_{s}\right)
$$

with $\zeta_{s}=\left(V_{s} / T_{e}\right)^{1 / 2}$. Hence $f_{c 2}$ is given by

$$
\begin{aligned}
f_{c 2} & =\frac{n_{c 2}}{\operatorname{erfc}\left(\zeta_{s}\right)}\left(\frac{m}{2 \pi e T_{e}}\right)^{3 / 2} \mathrm{e}^{-m v_{2}^{2} / 2 e T_{e}} ; \quad\left|v_{z 2}\right|>v_{s} \\
& =0 ; \quad \text { otherwise. }
\end{aligned}
$$

The ionization rate coefficient is found by integrating $\sigma_{\mathrm{iz}} v f_{c 2}$ over spherical coordinates $\left(v, \theta^{\prime}, \phi^{\prime}\right)$ in velocity space. The $\phi^{\prime}$ integration yields the factor $2 \pi$. We then obtain

$$
K_{\mathrm{izc}}=\frac{2 \pi}{n_{c 2}} \int_{0}^{\infty} \mathrm{d} v \int_{0}^{\pi} \sin \theta^{\prime} \mathrm{d} \theta^{\prime} v^{2} \cdot \sigma_{\mathrm{iz}} v \cdot f_{c 2}(v) .
$$

Substituting (C5) into (C6), we obtain

$$
\begin{aligned}
K_{\mathrm{izc}} & =\frac{4 \pi}{\operatorname{erfc}\left(\zeta_{s}\right)}\left(\frac{m}{2 \pi e T_{e}}\right)^{3 / 2} \\
& \times \int_{v_{s}}^{\infty} v^{2} \mathrm{~d} v \cdot \sigma_{\mathrm{iz}}(v) v \cdot \mathrm{e}^{-m v^{2} / 2 e T_{e}} \cdot \int_{0}^{\theta} \sin \theta^{\prime} \mathrm{d} \theta^{\prime},
\end{aligned}
$$

where $\cos \theta=v_{s} / v$. The $\theta^{\prime}$ integration yields the factor $\left(1-v_{s} / v\right)$. Inserting this into (C7) yields

$$
\begin{aligned}
K_{\mathrm{izc}} & =\frac{4 \pi}{\operatorname{erfc}\left(\zeta_{s}\right)}\left(\frac{m}{2 \pi e T_{e}}\right)^{3 / 2} \\
& \times \int_{v_{s}}^{\infty} v^{2} \mathrm{~d} v \cdot \sigma_{\mathrm{iz}}(v) v\left(1-\frac{v_{s}}{v}\right) \cdot \mathrm{e}^{-m v^{2} / 2 e T_{e}} .
\end{aligned}
$$

Making the substitution $\mathcal{E}=m v^{2} / 2 e$, we obtain the final result

$$
K_{\mathrm{izc}}=\left(\frac{8 e T_{e}}{\pi m}\right)^{1 / 2} \frac{1}{T_{e}^{2} \operatorname{erfc}\left(\zeta_{s}\right)} \mathcal{I}\left(V_{s}, T_{e}\right)
$$

where

$$
\mathcal{I}=\int_{V_{s}}^{\infty} \mathrm{d} \mathcal{E} \mathcal{E}\left[1-\left(\frac{V_{s}}{\mathcal{E}}\right)^{1 / 2}\right] \sigma_{\text {iz }}(\mathcal{E}) \mathrm{e}^{-\mathcal{E} / T_{e}} .
$$

For $V_{s} \leqslant 50 \mathrm{~V}$, the integration in (C10) is done numerically using the ionization cross section given in [14], and the results for $K_{\mathrm{izc}}$ are plotted in figure $\mathrm{C} 1$ versus $T_{e}$ over this range of $V_{s}$. Above $50 \mathrm{~V}$, the accelerated electrons are nearly beam-like, and we use the simpler expression $K_{\mathrm{izc}}=\sigma_{\mathrm{iz}}\left(2 e V_{s} / m\right)^{1 / 2}$. We see from the figure that as $V_{s}$ increases, the rate coefficient increases, with the greatest increase at lower temperatures. At $V_{s} \approx 300 \mathrm{~V}, K_{\text {izc }}$ has a maximum value of $2.1 \times 10^{-13} \mathrm{~m}^{3} \mathrm{~s}^{-1}$, and then falls slightly for still larger values of $V_{s}$. For $V_{s} \leqslant 50 \mathrm{~V}$, at each value of $V_{s}$, we determine the coefficients $A, B$ and $C$ that give the best fit to the rate coefficient

$$
K_{\mathrm{izc}}=A T_{e}^{B} \mathrm{e}^{-C / T_{e}}
$$

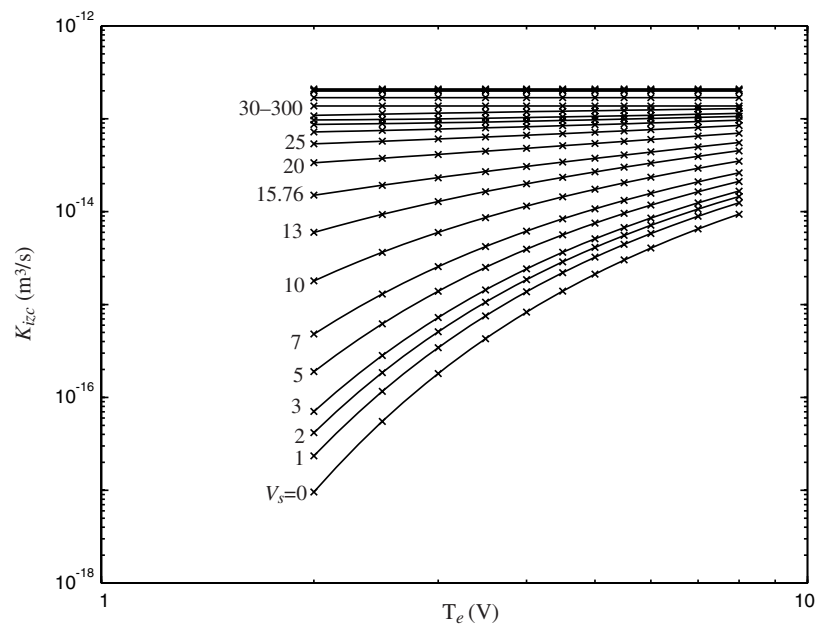

Figure C1. Upstream ionization rate coefficient $K_{\mathrm{izc}}$ versus $T_{e}$ at various double layer voltages $V_{s}$; the solid lines are the fits to (C11) using the $A B C$ coefficients given in table $\mathrm{C} 1$.

Table C1. Best fit values $A, B$ and $C$ for $K_{\text {izc }}$ in equation (C11).

\begin{tabular}{ccll}
\hline$V_{s}(\mathrm{~V})$ & $A\left(10^{-14} \mathrm{~m}^{3} \mathrm{~s}^{-1}\right)$ & $B$ & $C(\mathrm{~V})$ \\
\hline 0 & 2.1779 & 0.5735 & 16.256 \\
1 & 2.9091 & 0.4895 & 14.927 \\
2 & 3.1033 & 0.4701 & 13.879 \\
3 & 3.1693 & 0.4633 & 12.859 \\
5 & 3.0916 & 0.4691 & 10.844 \\
7 & 2.8508 & 0.4916 & 8.8487 \\
10 & 2.3443 & 0.5484 & 5.9006 \\
13 & 1.8176 & 0.6257 & 3.0954 \\
15.8 & 1.5217 & 0.6807 & 0.97202 \\
20 & 2.1201 & 0.5666 & -0.13575 \\
25 & 3.6450 & 0.3910 & -0.23700 \\
30 & 5.1994 & 0.2821 & -0.26627 \\
35 & 6.2641 & 0.2340 & -0.33219 \\
40 & 7.0458 & 0.2121 & -0.34701 \\
50 & 10.638 & 0.09751 & 0.089166 \\
70 & 13.755 & 0 & 0 \\
100 & 16.909 & 0 & 0 \\
200 & 20.078 & 0 & 0 \\
300 & 20.979 & 0 & 0 \\
500 & 20.121 & 0 & 0 \\
\hline
\end{tabular}

Note. $T_{e}$ is in the range $2-8 \mathrm{eV}$; cross section from [14].

over the range $2 \leqslant T_{e} \leqslant 8 \mathrm{~V}$. Above $50 \mathrm{~V}$, we use $A=K_{\text {izc }}$ with $B=C=0$. The best fit values are given in table $\mathrm{C} 1$.

\section{References}

[1] Raadu M A 1989 Phys. Rep. 17825

[2] Charles C and Boswell R W 2003 Appl. Phys. Lett. 821356

[3] Cohen S A, Siefert N S, Stange S, Boivin R F, Scime E E and Levinton F M 2003 Phys. Plasmas 102593

[4] Sun X et al 2005 Phys. Rev. Lett. 95025004

[5] Fruchtman A 2006 Phys. Rev. Lett. 96065002

[6] Boswell R W, Marsch E and Charles C 2006 Astrophys. J. 640 L199

[7] Charles C and Boswell R W 2003 Appl. Phys. Lett. 821356

[8] Langmuir I 1929 Phys. Rev. 33954

[9] Andrews J G and Allen J E 1971 Proc. R. Soc. Lond. A 320459

[10] Bohm D 1949 The Characteristics of Electrical Discharges in Magnetic Fields ed A Guthrie and R K Wakerling (New York: McGraw-Hill) chapter 3, p 77 
[11] Chan C, Hershkowitz N and Payne G L 1981 Phys. Lett. A 83328

[12] Lieberman M A and Lichtenberg A J 2005 Principles of Plasma Discharges and Materials Processing 2nd edn (New York: Wiley)
[13] Sutherland O, Charles C, Plihon N and Boswell R W 2005 Phys. Rev. Lett. 95205002

[14] www.kinema.com

[15] Charles C 2005 Phys. Plasmas 12044508 